\title{
SATELLITE GRAVITATIONAL ORBITAL PERTURBATIONS AND THE GRAVITOMAGNETIC CLOCK EFFECT
}

\author{
LORENZO IORIO \\ Dipartimento Interateneo di Fisica dell' Università di Bari, Via Amendola 173 \\ Bari, 70126, Italy
}

\begin{abstract}
In order to detect the gravitomagnetic clock effect by means of two counter-orbiting satellites placed on identical equatorial and circular orbits around the Earth with radius $7000 \mathrm{~km}$ their radial and azimuthal positions must be known with an accuracy of $\delta r=$ $10^{-1} \mathrm{~mm}$ and $\delta \phi=10^{-2}$ mas per revolution. In this work we investigate if the radial and azimuthal perturbations induced by the dynamical and static parts of the Earth' $\mathrm{s}$ gravitational field meet these requirements. While the radial direction is affected only by harmonic perturbations with periods up to some tens of days, the azimuthal location is perturbed by a secular drift and very long period effects. It results that the present level of accuracy in the knowledge both of the Earth solid and ocean tides, and of the static part of the geopotential does not allow an easy detection of the gravitomagnetic clock effect at least by using short arcs only.
\end{abstract}

\section{Introduction}

Latest years have seen great efforts, both from a theoretical and an experimental point of view, devoted to the measurement of the general relativistic Lense-Thirring effect $^{1,2}$ in the weak gravitational field of the Earth by means of artificial satellites.

Among the satellite-based experiments recently proposed, one of the most interesting is devoted to the detection of the the gravitomagnetic clock effect ${ }^{3,4,5}$. It consists in the fact that two clocks moving along pro- and retrograde circular equatorial orbits, respectively, about the Earth exhibit a difference in their proper times which, if calculated after some fixed angular interval, say $2 \pi$, amounts to:

$$
\left(\tau_{+}-\tau_{-}\right)_{\phi=2 \pi} \simeq 4 \pi \frac{J_{\oplus}}{M_{\oplus} c^{2}} \simeq 10^{-7} s
$$

where $J_{\oplus}$ and $M_{\oplus}$ are the rotational angular momentum and the mass, respectively, of the Earth; $c$ is the speed of light in vacuo. $\mathrm{In}^{4,6}$ it has been shown that for an orbit radius of $7000 \mathrm{~km}$ the radial and azimuthal locations of the satellites must be known at a level of accuracy of $\delta r \leq 10^{-1} \mathrm{~mm}$ and $\delta \phi \leq 10^{-2}$ mas per revolution. 
However, the studies conducted up to now on the feasibility and the error budget of such an experiment are still preliminary ${ }^{4,6}$.

In this paper we shall investigate in a quantitative manner the systematic errors induced on the radial and azimuthal locations by the Earth solid and ocean tidal perturbations and by the static part of the geopotential. The paper is organized as follows. In Section 2 and 3 the radial and azimuthal perturbations, respectively, induced by the most relevant tidal constituents are investigated. In Section 4 the radial and azimuthal perturbations generated by the static part of the geopotential are worked out. Section 5 is devoted to the conclusions.

\section{The radial error induced by the Earth solid and ocean tides}

According to ${ }^{7,8}$, the position perturbations in the radial direction can be expressed in general as:

$$
\Delta r=\sqrt{\Delta a^{2}+\frac{1}{2}\left[(e \Delta a+a \Delta e)^{2}+(a e \Delta \mathcal{M})^{2}\right]},
$$

where $a, e$ and $\mathcal{M}$ denote the satellite' s semimajor axis, eccentricity and mean anomaly. In eq.(2) the perturbation amplitudes are the rss values of the perturbations and small eccentricity approximations have been extensively applied.

Since the difference in the proper orbital periods to be investigated is integrated over $2 \pi$ with respect to the azimuthal angle $\phi$, as viewed by an inertial observer fixed with the distant quasars, we shall consider only the long period perturbations averaged over an orbital revolution. This is accomplished by assuming those values for the indices $l, p, q^{9,10}$ which satisfy the relation $l-2 p+q=0$. Since the tidal perturbations on the semimajor axis $a$ are proportional just to $l-2 p+q$, all the terms in $(\Delta a)^{2}$ and $\Delta a$ of eq.(2) vanish and it reduces to:

$$
\Delta r=\sqrt{\frac{1}{2}\left[(a \Delta e)^{2}+(a e \Delta \mathcal{M})^{2}\right]} .
$$

For $e=0$ eq.(3) becomes:

$$
\Delta r_{\text {tides }}=\frac{1}{\sqrt{2}} a \Delta e_{\text {tides }} .
$$

For a constituent characterized by a given set of indices $\{l, m, p, q\}$, the first order tidal perturbation amplitude for the eccentricity turns out to be*

$$
\Delta e_{l m p q} \propto-\frac{(l-2 p) F_{l m p} G_{l p q}}{e},
$$

where $F_{l m p}$ and $G_{l p q}$ are the inclination and the eccentricity functions, respectively, as can be found $\mathrm{in}^{12}$.

*This holds for $l-2 p+q=0$. Note that for the eccentricity there are no second order, indirect perturbations due to the oblateness of the $\operatorname{Earth}^{9,10,11}$, contrary to the node, the perigee and the mean anomaly. 
Eq.(5) allows to obtain a preliminary insight into those perturbations which, for a given set of indices $l, m, p, q$, vanish. Note that, for $e=0$, eq.(5) could become singular. Concerning this problem it must be considered that, since $G_{l p q} \propto e^{|q|}$, the behaviour of $\frac{G_{l p q}}{e} \propto e^{|q|-1}$ is crucial. If $|q|-1 \equiv k>0$, i. e. $q>1$ or $q<-1$, then for a circular orbit $\frac{G_{l p q}}{e}=0$ and the perturbation vanishes. If $|q|-1=0$, i.e. $q= \pm 1$, then $\frac{G_{l p q}}{e}=$ const. Problems may arise only if $q=0$, but we shall see that, in general, in the cases in which $q$ takes such value, $l-2 p=0$ also holds so that the perturbations identically vanish with no regards to the eccentricity or the inclination of the satellite.

Let us start with the tidal perturbations of even degree. For $l=2 n, p=0, \ldots, l$ and $l-2 p+q=0$, the allowable values for q satisfy the above stated conditions so that we can conclude that there are no radial tidal perturbations of even degree. Since for the solid Earth tides we consider only the $l=2$ constituents, this result rules out their possible influence on the radial error budget in the gravitomagnetic clock experiment.

Now we shall consider the odd degree case. For $l=3$ there are no problems because $q$ never vanishes. Moreover, for $p=1, q=-1$ and $p=2, q=1 \frac{G_{l p q}}{e}=1$ and $l-2 p= \pm 1$ while for the other sets of indices the perturbations vanish because $\frac{G_{l p q}}{e}=0$. When we consider the inclination functions corresponding to the indices for which $\frac{G_{l p q}}{e}$ and $l-2 p$ differ from zero, i. e. $F_{3 m 1}$ and $F_{3 m 2}$, and evaluate them for $i=0$ we find that only $F_{311}(i=0)=-\frac{3}{2}$.

So we can conclude that the radial direction is perturbed only by the $l=3, m=$ $1, p=1, q=-1$ ocean tides.

The full expression for the eccentricity perturbation amplitude due to ocean tides $^{9,10}$, in our case, is given by

$$
\Delta e_{l m p q f}=\frac{4 \pi G \rho_{w}}{n a \dot{\gamma}_{l m p q f}^{+}}\left(\frac{R_{\oplus}}{a}\right)^{l+2}\left(\frac{1+k_{l}^{\prime}}{2 l+1}\right) C_{l m f}^{+}\left[-\frac{(l-2 p) F_{l m p} G_{l p q}}{e}\right],
$$

where:

- $G=6.67259 \cdot 10^{-8} \mathrm{~cm}^{3} \mathrm{~g}^{-1} \mathrm{~s}^{-2}$ is the Newtonian gravitational constant ${ }^{14}$

- $n=\sqrt{\frac{G M_{\oplus}}{a^{3}}}$ is the satellite mean motion; $G M_{\oplus}=3.986 \cdot 10^{20} \mathrm{~cm}^{3} \mathrm{~s}^{-2} 14$

- $\rho_{w}=1.025 \mathrm{~g} \mathrm{~cm}^{-3}$ is the water density ${ }^{14}$

- $R_{\oplus}=6378 \cdot 10^{5} \mathrm{~cm}$ is the Earth' s equatorial radius

- $k_{l}^{\prime}$ is the load Love number. $k_{2}^{\prime}=-0.3075, k_{3}^{\prime}=-0.1950^{15,16}$

- $C_{l m f}^{+}, \mathrm{cm}$ is the ocean tidal height as can be found in EGM96 model ${ }^{17}$

- $\dot{\gamma}_{l m p q f}^{+}=(l-2 p) \dot{\omega}+(l-2 p+q) \mathcal{M}+m \dot{\Omega}+\left(j_{2}-m\right) \dot{s}+j_{3} \dot{h}+j_{4} \dot{p}+j_{5} \dot{N}^{\prime}+j_{6} \dot{p}_{s}$ in which $\omega$ and $\Omega$ are the satellite' s perigee and node and the integers $j_{i}, i=2, . .6$ refer to the Doodson number ${ }^{18,19}$ by which each tidal constituent is classified. For the astronomical longitudes $s, \ldots p_{s}$ see, e.g. ${ }^{19}$. Recall that $\dot{\gamma}_{l m p q f}^{+}$has to be evaluated on the chosen reference orbit.

\footnotetext{
${ }^{\dagger}$ Here we shall consider only the prograde waves ${ }^{13}$.
} 
For $l=3, m=1, p=1, q=-1$, and by putting $\dot{\gamma}_{l m p q f}^{+}=\frac{2 \pi}{P_{p e r t}}$, eq.(4) becomes:

$$
\Delta r_{311-1 f}=\left(8.80 \cdot 10^{25} \mathrm{~cm}^{7 / 2} \mathrm{~s}^{-1}\right) \times a^{-7 / 2} \times P_{\text {pert }} \times C_{l m f}^{+} .
$$

Among the tesseral tides, the $K_{1}(165.555)$ is by far the most important in perturbing the near Earth satellites' orbits ${ }^{20,21}$. So it seems reasonable to calculate eq.(7) for it in order to obtain an upper bound in the order of magnitude of the tidally induced perturbations on $\Delta r$. For such a constituent:

$$
C_{31}^{+}\left(K_{1}\right)=0.95 \mathrm{~cm}
$$

and $\dot{\gamma}_{311-1}^{+}\left(K_{1}\right)=\dot{\omega}+\dot{\Omega}$. If we assume as reference orbit a secularly precessing ellipse $^{12}$, we obtain:

$$
P_{\text {pert }}=-\frac{4 \pi}{3} \frac{1}{C_{2,0} R_{\oplus}^{2} \sqrt{G M_{\oplus}}} a^{7 / 2}=\left(4.7639 \cdot 10^{-25} \mathrm{~cm}^{-7 / 2} s\right) \times a^{7 / 2} .
$$

Eqs.(7)-(9) tell us the important feature that for $K_{1}, l=3, m=1, p=1, q=-1$ the perturbation amplitude is independent from the satellite' s semimajor axis. For $a=7000 \mathrm{~km}$ and $C_{2,0}=-J_{2}=-0.00108261$ we obtain $P_{\text {pert }}=50$ days. By using eqs.(8)-(9) in eq.(17) we obtain:

$$
\Delta r_{311-1}\left(K_{1}\right)=39.849 \mathrm{~cm} .
$$

According to EGM96 model ${ }^{17}$, the percentage error on $C_{31}^{+}\left(K_{1}\right)$ amounts to $5.2 \%$; this yields $\delta r_{311-1}\left(K_{1}\right) \simeq 2.07 \mathrm{~cm}$. Despite the amplitude of this long period mismodeled perturbation is 2 orders of magnitude greater than the maximum allowable error $\delta r_{\max }=1 \cdot 10^{-1} \mathrm{~mm}$, it must be noted that its period $P_{\text {pert }}$ amounts to only 50 days. This implies that if an observational time span $T_{o b s}$ which is an integer multiple of $P_{\text {pert }}$, i. e. some months, is adopted the tidal perturbative action of $K_{1}$ can be averaged out.

\section{The azimuthal error induced by the Earth solid and ocean tides}

Concerning the angular variable which defines the position of the satellite on the orbit, for an equatorial, circular orbit it seems reasonable to adopt for its rate of change:

$$
\dot{\phi}=\dot{\omega}+\dot{\Omega} \cos i+\dot{\mathcal{M}}
$$

In it $\dot{\omega}+\dot{\Omega} \cos i$ represents an angular velocity around the direction of the orbital angular momentum ${ }^{22}$; it is valid for any inclination angle $i$. In order to account for the fact that the orbit is circular we add to it $\dot{\mathcal{M}}$. See also ${ }^{23}$. About the perturbations on the latter Keplerian orbital element, it turns out that ${ }^{22}$ in $\Delta \mathcal{M}$ one has to consider also the indirect perturbations on the mean motion $n$ due to the cross coupling with the semimajor axis $a$. Since they are proportional to $l-2 p+q$, they vanish when only long period perturbations are considered, as is the case here. 
The perturbation amplitudes on the node, the perigee and the mean anomaly are proportional toł

$$
\begin{gathered}
\Delta \Omega_{l m p q f} \propto \frac{1}{\sqrt{1-e^{2}} \sin i} G_{l p q} \frac{d F_{l m p}}{d i} \\
\Delta \omega_{l m p q f} \propto \frac{\sqrt{1-e^{2}}}{e} \frac{d G_{l p q}}{d e} F_{l m p}-\frac{\cos i}{\sqrt{1-e^{2}} \sin i} G_{l p q} \frac{d F_{l m p}}{d i}, \\
\Delta \mathcal{M}_{l m p q f} \propto-\frac{1-e^{2}}{e} \frac{d G_{l p q}}{d e} F_{l m p}+2(l+1) F_{l m p} G_{l p q} .
\end{gathered}
$$

By assuming $1-e^{2}=\sqrt{1-e^{2}} \simeq 1$ for $e \rightarrow 0$, with the aid of eqs.(11)-(14) we obtain:

$$
\Delta \phi_{l m p q f} \propto 2(l+1) F_{l m p} G_{l p q} .
$$

As already done in the previous section, eq.(15) can be used in order to forecast which perturbations will vanish.

For $l=2$ only the combination $l=2, p=1, q=0$ yields a nonzero eccentricity function: $G_{210}=\left(1-e^{2}\right)^{-3 / 2}=1$. Among the corresponding inclination functions $F_{2 m 1}$, for $i=0$ we have $F_{201}=-1 / 2$. The same conclusion holds also for $l=4$ with $G_{420}(e=0)=1$ and $F_{402}(i=0)=3 / 8$. So we can conclude that for $l=2,4$ only the zonal tides, both solid and ocean, cause nonvanishing perturbations on the satellite's azimuthal variable.

Concerning the odd degree perturbations, they all vanish since for $l=3,5, q$ is always nonzero, so that, since $G_{l p q} \propto e^{|q|}$, for circular orbits all the eccentricity functions vanish. The conclusion is that the odd part of the ocean tidal spectrum does not induce systematic errors on the satellite's azimuthal variable.

For a given constituent of degree $l$, order $m$ and frequency $f$ the full expressions for the solid Earth and ocean tidal perturbation amplitude (progressive waves only) are, respectively:

$$
\begin{aligned}
\Delta \phi_{l m p q f}^{\text {solid }} & =\frac{g}{n a^{2} \dot{\gamma}_{l m p q f}}\left(\frac{R_{\oplus}}{a}\right)^{l+1} A_{l m} k_{l m f}^{(0)} H_{l}^{m}\left[2(l+1) F_{l m p} G_{l p q}\right] \\
\Delta \phi_{l m p q f}^{\text {ocean }} & =\frac{4 \pi G \rho_{w}}{n a \dot{\gamma}_{l m p q f}^{+}}\left(\frac{R_{\oplus}}{a}\right)^{l+2}\left(\frac{1+k_{l}^{\prime}}{2 l+1}\right) C_{l m f}^{+}\left[2(l+1) F_{l m p} G_{l p q}\right]
\end{aligned}
$$

where:

- $g=978.0327 \mathrm{~cm} \mathrm{~s}^{-2}$ is the acceleration of gravity at the surface of the Earth as if it was perfectly spherical ${ }^{14}$

- $\dot{\gamma}_{l m p q f}=\dot{\gamma}_{l m p q f}^{+}$

- $A_{l m}=\sqrt{\frac{2 l+1}{4 \pi} \frac{(l-m) !}{(l+m) !}}$

- $k_{l m f}^{(0)}$ is the Love number for the free space potential ${ }^{14,24}$

- $H_{l}^{m}$ are the Doodson coefficients with a different normalization ${ }^{14,25}$

$\ddagger$ The second order, indirect perturbations will not be considered here since it can be demonstrated that they vanish in this case. 
We shall start by considering the three most relevant $l=2 m=0$ zonal tides:

- 18.6 - year $(055.565) ; P_{\text {pert }}=6798.38$ days; $k_{20}^{(0)}=0.315 ; H_{2}^{0}=2.792 \mathrm{~cm}$

- 9.3 -year $(055.575) ; P_{\text {pert }}=3399.19$ days $; k_{20}^{(0)}=0.313 ; H_{2}^{0}=2.72 \cdot 10^{-2} \mathrm{~cm}$

- $S_{a}(056.554) ; P_{\text {pert }}=365.27$ days $; k_{20}^{(0)}=0.307 ; H_{2}^{0}=-4.92 \cdot 10^{-1} \mathrm{~cm} ; k_{2}^{\prime}=$ $-0.3075 ; C_{l m f}^{+}=2.54 \mathrm{~cm}$

For $l=2$ eqs.(16)-(17) becomes:

$$
\begin{gathered}
\Delta \phi^{\text {solid }}=\left(-3.77 \cdot 10^{18} \mathrm{~cm}^{5 / 2} \mathrm{~s}^{-1}\right) \times a^{-7 / 2} \times k_{20}^{(0)} \times P_{\text {pert }} \times H_{2}^{0}, \\
\Delta \phi^{\text {ocean }}=\left(-4.707 \cdot 10^{17} \mathrm{~cm}^{5 / 2} \mathrm{~s}^{-1}\right) \times a^{-7 / 2} \times P_{\text {pert }} \times C_{l m f}^{+} .
\end{gathered}
$$

Note that, since for the $l=2$ zonal tides $P_{\text {pert }}$ does not depend on the satellite' s semimajor axis but only on the astronomical arguments, $\Delta \phi_{l m p q f}$ depends on the orbit's radius through $a^{-7 / 2}$, contrary to $\Delta r\left(K_{1}\right)$, as shown in the previous Section. For $a=7000 \mathrm{~km}$ we have:

- $\Delta \phi(18.6-$ year $)=-4.431 \cdot 10^{4}$ mas

- $\Delta \phi(9.3-$ year $)=-214.4$ mas

- $\Delta \phi\left(S_{a}\right)=408$ mas (solid); 857.6 mas (oceanic)

The zonal tidal perturbations on the satellite's azimuthal location are particularly insidious not only because their nominal amplitudes are up to 6 orders of magnitude greater than the maximum allowable error $\delta \phi_{\max }=10^{-2}$ mas, but also because they have periods very long, so that there is no hope they average out on reasonable $T_{o b s}$. Concerning the 18.6-year tide, by assuming an uncertainty of $1.5 \%$ on $k_{20}^{(0)} 20$, the mismodeling on its perturbation amounts to -664 mas which is, however, very far from $\delta \phi_{\max }$.

\section{Static geopotential perturbations}

As can be found $\mathrm{in}^{12}$, the perturbing function of degree $l$ and order $m$ of the static part of the geopotential can be cast into the form:

$$
V_{l m}=\frac{G M_{\oplus} R_{\oplus}^{l}}{a^{l+1}} F_{l m p} G_{l p q} S_{l m p q}
$$

where:

- $S_{l m p q}=\left[\begin{array}{c}C_{l m} \\ -S_{l m}\end{array}\right]_{l-m \text { odd }}^{l-m \text { even }} \cos \psi_{l m p q}+\left[\begin{array}{c}S_{l m} \\ C_{l m}\end{array}\right]_{l-m \text { odd }}^{l-m \text { even }} \sin \psi_{l m p q}$

- $C_{l m}, S_{l m}$ are the unnormalized Stokes' geopotential coefficients ${ }^{17}$

- $\psi_{l m p q}=(l-2 p) \omega+(l-2 p+q) \mathcal{M}+m(\Omega-\theta)$ in which $\theta$ is the sidereal angle

Concerning the preliminary analysis of the even degree perturbations, the same conclusions of the previous sections hold. All the perturbations on the radial directions vanish, $\Delta r_{\text {static }}=0$, while for the satellite's azimuthal location only the zonal contributions are to be considered. Let us work out explicitly the perturbation due to the main even zonal coefficient $C_{2,0}$. For the precessional secular rates induced by it of a satellite on circular orbit we have:

$$
\frac{d \omega}{d t}=\frac{3}{4} \frac{n C_{2,0} R_{\oplus}}{a^{2}}\left(1-5 \cos i^{2}\right)
$$




$$
\begin{gathered}
\frac{d \Omega}{d t}=\frac{3}{2} \frac{n C_{2,0} R_{\oplus}}{a^{2}} \cos i, \\
\frac{d \mathcal{M}}{d t}=-\frac{3}{4} \frac{n C_{2,0} R_{\oplus}}{a^{2}}\left(3 \cos i^{2}-1\right) .
\end{gathered}
$$

By inserting eqs.(21)-(23), evaluated for $\mathrm{i}=0$, into eq.(11) we obtain:

$$
\frac{d \phi}{d t}=-3 \sqrt{G M_{\oplus}} C_{2,0} R_{\oplus}^{2} a^{-7 / 2}=\left(2.637 \cdot 10^{25} \mathrm{~cm}^{7 / 2} s^{-1}\right) \times a^{-7 / 2} .
$$

For a circular orbit of radius $7000 \mathrm{~km}$ the azimuthal secular rate is:

$$
\frac{d \phi}{d t}=1.89 \cdot 10^{10} \text { mas } / y \text {. }
$$

It is important to evaluate the error induced on such rate by the poor knowledge of the Earth' s gravitational field. According to the EGM96 model ${ }^{17}$, a relative uncertainty of $7.3 \cdot 10^{-8}$ weighs on the $C_{2,0}$ coefficient. This yields $\delta \dot{\phi} \simeq 1380 \mathrm{mas} / \mathrm{y}$, which is equivalent to $\delta \phi \simeq 2.5 \cdot 10^{-1}$ mas per revolution, being $P_{\text {orb }}=\frac{2 \pi}{n}=5.82 \cdot 10^{3}$ $\mathrm{s}$ for $a=7000 \mathrm{~km}$. Such error is 1 order of magnitude greater than $\delta \phi_{\max } \simeq 10^{-2}$ mas.

Concerning the perturbations of odd degree, let us consider in detail the most important geopotential harmonic of degree $l=3$. By reasoning as in the previous sections, it results that the satellite' $\mathrm{s}$ azimuthal location is not perturbed by the $l=3$ part of the geopotential spectrum. Concerning $\Delta r$, the perturbation corresponding to the combination $l=3, m=1, p=1, q=-1$ does not vanish. The full expression for the geopotential perturbation on the eccentricity is given by:

$$
\Delta e_{l m p q}=\frac{G M_{\oplus}}{n a^{2} \dot{\psi}_{l m p q}}\left(\frac{R_{\oplus}}{a}\right)^{l} \frac{G_{l p q}}{e} F_{l m p}[-(l-2 p)] S_{l m p q}
$$

In our case it yields:

$$
\Delta r_{\text {static }}=\frac{3}{2 \sqrt{2}} \sqrt{G M_{\oplus}} R_{\oplus}^{3} a^{-7 / 2} \times \frac{P_{\text {pert }}}{2 \pi} \times\left[C_{3,1} \cos \psi_{311-1}+S_{3,1} \sin \psi_{311-1}\right],
$$

with:

$$
\dot{\psi}_{311-1} \equiv \frac{2 \pi}{P_{\text {pert }}}=\dot{\omega}+\dot{\Omega}-\dot{\theta}
$$

With $a=7000 \mathrm{~km}$ we have for the period and the amplitudes of the harmonic terms:

$$
\begin{gathered}
P_{\text {pert }}=8.81 \cdot 10^{4} \mathrm{~s} \\
A_{\Delta r}=\left(-8.481 \cdot 10^{9} \mathrm{~cm}\right) \times\left[\begin{array}{l}
C_{3,1} \\
S_{3,1}
\end{array}\right] .
\end{gathered}
$$

According to ${ }^{17}$ the mismodeling weighing on the Stokes' coefficients of interest amounts to $\delta C_{3,1}=1.5 \cdot 10^{-10}, \delta S_{3,1}=1.3 \cdot 10^{-10}$. This leads to a mismodeled radial perturbation:

$$
\delta r_{\text {static }} \simeq(1.3 \mathrm{~cm}) \cos \psi_{311-1}+(1.15 \mathrm{~cm}) \sin \psi_{311-1}
$$


It results to be 2 orders of magnitude greater than the allowable $\delta r_{\max } \simeq 10^{-1} \mathrm{~mm}$. However, it must be pointed out that such a mismodeled perturbation averages out on an observational time span $T_{\text {obs }}$ which is an integer multiple of 1 day, its period amounting to $24.48 \mathrm{hr}$. The same conclusions can be drawn for the other higher odd degree nonvanishing radial perturbations.

\section{Discussion and conclusions}

In this paper we have explicitly calculated, by averaging over one orbital revolution, the most relevant perturbations $\Delta r$ and $\Delta \phi$ due to the dynamical and static part of the Earth's gravitational field on the radial and azimuthal locations of a satellite placed in an equatorial, circular orbit with radius of $7000 \mathrm{~km}$. Furthermore, we have compared the mismodeling induced on such perturbations by the poor knowledge of the parameters of the Earth' s gravitational field to the maximum errors per revolution $\delta r_{\max } \simeq 10^{-1} \mathrm{~mm}$ and $\delta \phi_{\max } \simeq 10^{-2}$ mas allowable in order to detect successfully the gravitomagnetic clock effect.

Concerning the radial direction, it is affected by harmonic perturbations induced by the odd degree part, both static and dynamical, of the Earth's gravitational field. If, from one hand, the related mismodeling is 2 orders of magnitude greater than $\delta r_{\max }$, from the other hand it must be pointed out that such mismodeled perturbations average out on not too long time spans since their periods range from 1 to 50 days.

The situation for the azimuthal angle is different. It is acted upon by the even degree zonal harmonics of the Earth's gravitational field. The zonal tides are very insidious since they induce perturbations with great amplitudes acting on very long periods, so that it is necessary to wait for several years in order to average out their mismodeled effect which are up to 4 orders of magnitude greater than $\delta \phi_{\max }$. The $l=2, m=0$ part of the geopotential induces also a secular drift on the azimuthal satellite' s angle; the uncertainties in $C_{2,0}$ induces on it a mismodeled rate per revolution which is 1 order of magnitude grater than $\delta \phi_{\max }$.

The conclusions outlined here hold for $r=7000 \mathrm{~km}$; let us see how the situation changes with different values for the orbital radius. The possible scenarios turn out to be very intricate. Indeed, from one hand we have secular or semisecular mismodeled perturbations which could be reduced only by enlarging the orbit radius, and from the other hand there are the periodic mismodeled perturbations whose periods grow with the orbit radius making so much more difficult to average them out on reasonable time spans. Moreover, it should also be considered that the maximum allowable errors depend on the orbit radius and they decrease with increasing radius putting, in this way, more stringent constraints on the mismodeled gravitational perturbations. This is shown in Fig.(11). In Fig.(2) and Fig.(3) we show how the mismodeled perturbative amplitudes due to the 18.6-year tide and the $C_{2,0}$ depend on the orbit radius. From an inspection of Fig.(1) and Fig.(2) it can be noted that the major problems come from the azimuthal error and the perturbation induced 

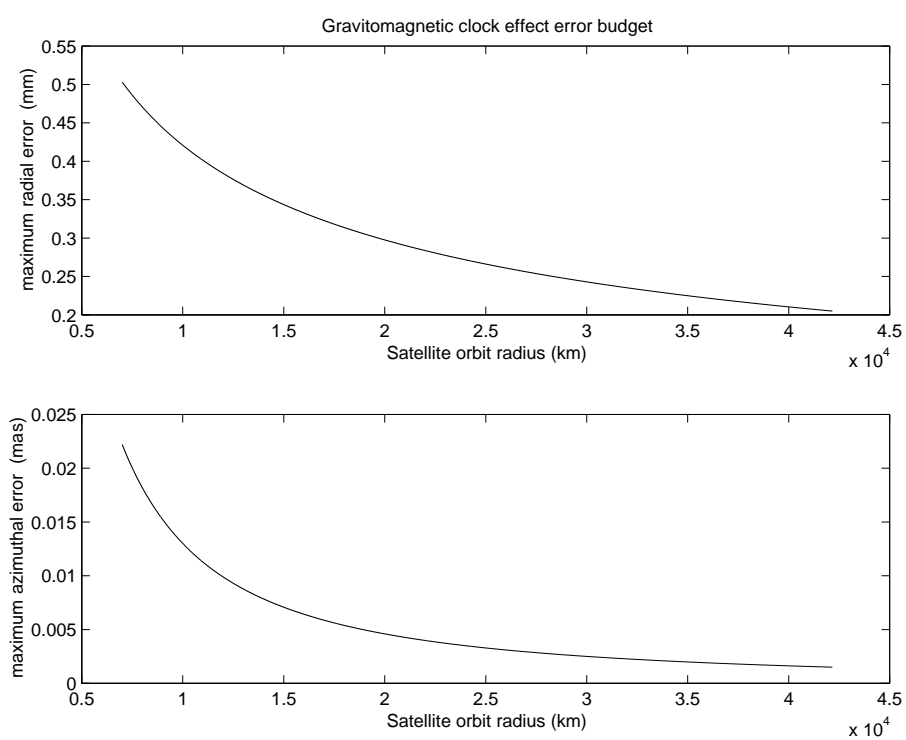

Fig. 1. Maximum allowable errors in the radial and azimuthal locations. The values for the orbit radius span from $7000 \mathrm{~km}$ to $42160 \mathrm{~km}$ for a geostationary satellite.

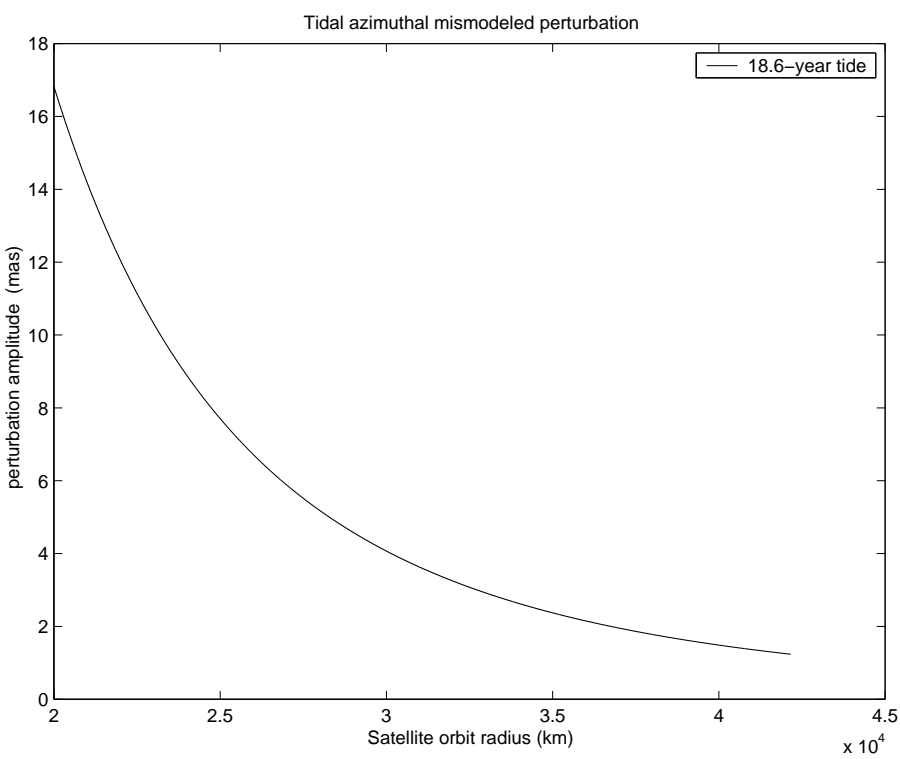

Fig. 2. Mismodeled azimuthal perturbation induced by the 18.6-year tide. 


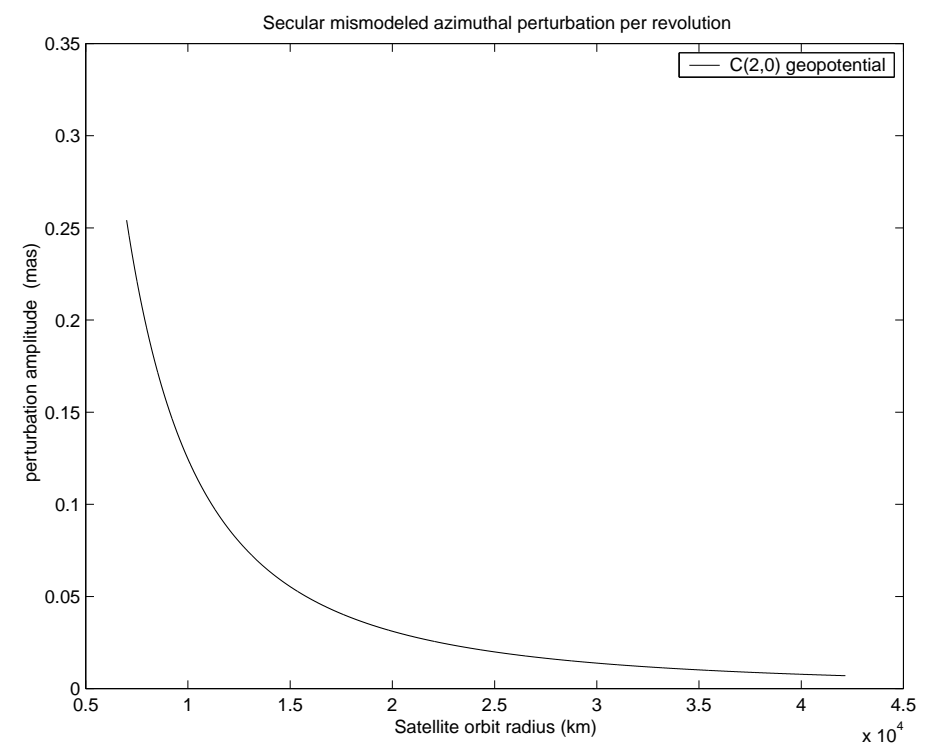

Fig. 3. Mismodeled azimuthal rate per revolution induced by the $C_{2,0}$ geopotential coefficient. The values for the orbit radius span from $7000 \mathrm{~km}$ to $42160 \mathrm{~km}$ for a geostationary satellite.

by the 18.6-year tide: as the orbit radius grows, the mismodeled tidal perturbation is always greater than the maximum allowable error by 2 or 3 orders of magnitude. Since this important source of systematic error cannot be made harmless by varying the orbital parameters of the satellites, it should be necessary to average out its effect: but this means to choose a time span $T_{o b s}=18.6$ years at least.

By inspecting Fig.(化) it can be noted the growth of the period of the radial tidal perturbation is induced by the $K_{1}, l=3 p=1 q=-1$. This is an important feature since its mismodeled amplitude is at $\mathrm{cm}$ level and is independent from the orbit radius. Moreover, $\delta r_{\max }$ is of the order of $10^{-1} \mathrm{~mm}$, so that we could eliminate the effect of such a perturbation only by averaging it over an integer multiple of its period.

We can conclude that the present level of accuracy in the knowledge both of the Earth solid and ocean tides, and of the static part of the geopotential does not allow an easy detection of the gravitomagnetic clock effect at least by using short arcs only.

\section{Acknowledgements}

I wish to thank B. Mashhoon for the useful material supplied to me and for his useful suggestions. I am also grateful to I. Ciufolini and E. Pavlis. Special thanks to L. Guerriero who supported me at Bari. 


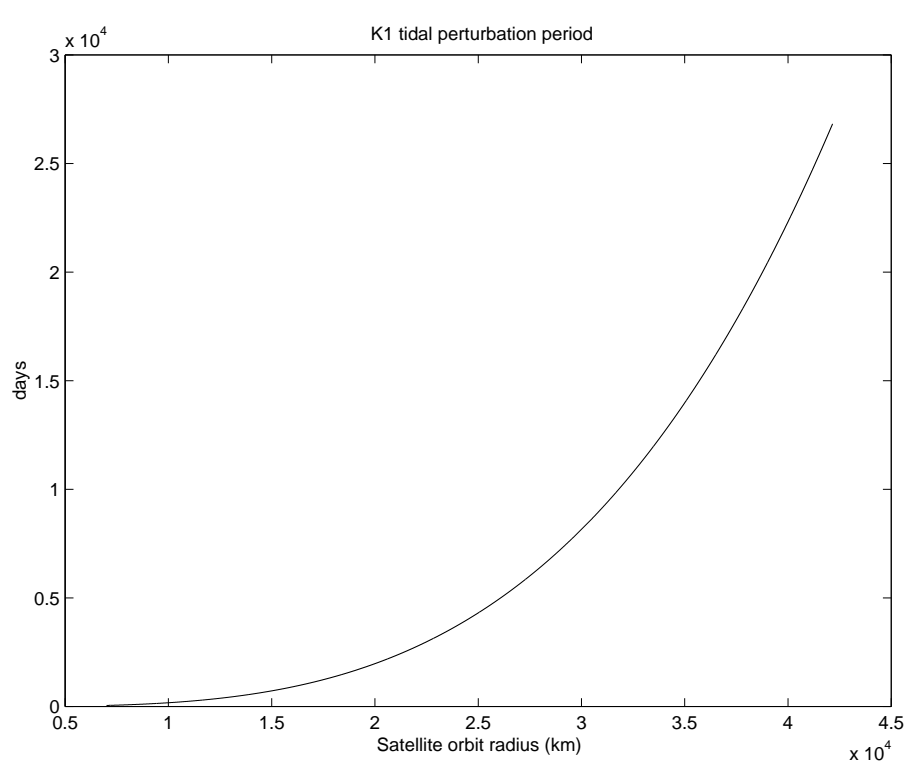

Fig. 4. Period of the $K_{1} l=3 p=1 q=-1$ radial tidal perurbation. The values for the orbit radius span from $7000 \mathrm{~km}$ to $42160 \mathrm{~km}$ for a geostationary satellite.

\section{References}

1. J. Lense and H. Thirring, Phys. Z. 19 (1918) 156, translated by B. Mashhoon, F. W. Hehl and D. S. Theiss, Gen. Rel. Grav. 16 (1984) 711.

2. I. Ciufolini and J. A. Wheeler, Gravitation and Inertia (Princeton University Press, Princeton, N. Y., 1995).

3. J. M. Cohen and B. Mashhoon, Physics Letters A181 (1993) 353.

4. H. I. M. Lichtenegger, F. Gronwald, and B. Mashhoon, Adv. Sp. Res. 25 (2000) 1255.

5. B. Mashhoon, F. Gronwald, and H. I. M. Lichtenegger, in Testing General Relativity In Space, edited by C. Lämmerzahl, G. W. F. Everitt and F. W. Hehl (Springer, Berlin, 2000); A. Tartaglia Gen. Rel. Grav. 32 (2000) 1745; A. Tartaglia Class. Quant. Grav. $17(2000) 783$.

6. F. Gronwald, E. Gruber, H. Lichtenegger, and R. A. Puntigam, Report No. ESA SP420, 1997.

7. R. G., Williamson and B. C. Douglas, Wolf Res. and Dev. Corp., Riverdale, Md., Report No. NAS-11726-163, 1972.

8. D. Christodoulidis, D. E. Smith, R. G. Williamson and S. M. Klosko, J. Geophys. Res. B6 (1988) 6216.

9. J. M. Dow, PhD thesis, München, 1988.

10. S. Casotto, PhD thesis, The University of Texas, 1989.

11. G. Balmino, in The Use of Artificial Satellites for Geodesy and Geodynamics, ed. G. Veis (National Technical University, Athens, 1974).

12. W. M. Kaula, Theory of Satellite Geodesy (Blaisdell Publishing Company, Waltham, 1966). 
13. E. W. Schwiderski, Rev. Geophys. Space Phys. 18 (1980) 243.

14. D. D. McCarthy, U. S. Naval Observatory, Report No. IERS Technical Note 21, 1996.

15. W. E. Farrell, Rev. Geophys. Space Phys. 10 (1972) 761.

16. S. D. Pagiatakis, Geophys. J. Int. 103 (1990) 541.

17. E. C. Pavlis et al., NASA, Report No. NASA/TP-1998-206861, 1998.

18. A. T. Doodson, Proc. R. Soc. A. 100 (1921) 305.

19. J. J. Dronkers, Tidal Computations (North-Holland Publishing Company, Amsterdam, 1964).

20. L. Iorio, submitted to Celest. Mech. 2000.

21. L. Iorio, submitted to Astron. and Geophys. 2000.

22. A. Milani, P. Farinella and A. M. Nobili, Non-Gravitational Perturbations and Satellite Geodesy (Adam Hilger, Bristol, 1989).

23. G. W. Rosborough and B. D. Tapley, Celest. Mech. 40 (1987) 409.

24. P. M. Mathews, B. A. Buffet, and I. I. Shapiro, Geophys. Res. Lett., 22 (1995) 579.

25. F. Roosbeek, Geophys. J. Int., 126 (1996) 197. 